\title{
Short review of aluminum hydroxide related lesions in preclinical studies and their relevance
}

\begin{abstract}
Aluminum is currently the most commonly used vaccine adjuvant. Toxicity and safety in regards to the use of aluminum adjuvants is highly controversial and also confused by conflicting study results. In general, it is regarded as safe by the pharmaceutical industry and the various regulatory agencies. Nevertheless, aluminum is a well demonstrated toxin in biological systems and its specific impacts on the nervous system have been widely documented. In regulatory preclinical studies, one of the co-authors has consistently identified the well-known local reaction granuloma. Except for granulomas at the injection site, other findings have not been observed in the course of the histopathologic evaluation. There were no indictors of neurotoxicity neither lesion in susceptible organs including the bone or immune system, nor indications for a general intoxication that could be related to exposure with aluminum hydroxide. However, other studies with various animal models have reported aluminum hydroxide to induce motor deficits, motor degeneration and neuro inflammation. A recent technology, taking advantage of fluorescent nano diamonds, that allows aluminum hydroxide particles to be traced in tissue have shown the progressive shrinkage of the local granuloma and the translocation of aluminum from the injection site to draining lymph nodes, spleen and brain tissue. Further it demonstrated differences between strains, doses, route of administration and injection site. Thus, these parameters should be taken into account in design for future preclinical models.
\end{abstract}

Keywords: vaccine, adjuvant, aluminum hydroxide, $\mathrm{al}(\mathrm{oh})^{3}$, preclinical, toxicity, gulf war syndrome, macrophagic myofasciitis, granulomas, neurotoxicity, th2, translocation
Volume 4 Issue 2 - 2017

Nils Warfving, Jürgen Laufs, Klaus Weber

AnaPath Services GmbH, Switzerland

Correspondence: Nils Warfving, AnaPath Services GmbH, Hammerstrasse 49, CH-44I 0 Liestal, Switzerland, Tel 0041619064000, Email nwarfving@anapath.ch

Received: January 27, 2017| Published: February 27, 2017

\section{Introduction}

Aluminum compounds were first identified as adjuvant over 90years ago. ${ }^{1}$ Adjuvants (immunopotentiators or immuno modulators) in vaccine formulations aim to enhance, accelerating and prolong the specific immune response towards the desired response to vaccine antigens. Advantages of adjuvants include the enhancement of the immunogenicity of antigens, modification of the nature of the immune response, the reduction of the antigen amount needed for a successful immunization, the reduction of the frequency of booster immunizations needed and an improved immune response in elderly and immuno compromised vaccines. ${ }^{2}$ Currently, aluminum is the most commonly used vaccine adjuvant and it is incorporated in vaccine formulas by various forms (aluminum hydroxide, aluminum phosphate and aluminum sulfate). ${ }^{1}$ The sum of officially scheduled vaccines deemed necessary for children in the U.S. has increased from 10 in the 1980s to 32 in the late $2000 \mathrm{~s}, 18$ of which contain aluminum adjuvants. Despite the longstanding and widespread use of aluminum adjuvants their precise mechanism of action remains poorly understood. ${ }^{3}$ The physiochemical mechanism can be described by aluminum hydroxide stimulation of the immune system by inducing the release of uric acid, an immunological danger signal, which strongly attracts certain types of monocytes who differentiate into dendritic cells. The antigen is carried by the dendrite cells to the lymph nodes where it stimulates $\mathrm{T}$ cells and B cells. ${ }^{4}$ Aluminum adjuvant is potent stimulators of the immune system and specially shift the immune response towards a Th2 profile. ${ }^{3}$

\section{Regulatory agencies}

Toxicity and safety in regards to the use of aluminum adjuvant is highly controversial and also confused by conflicting study results. In general, it is regarded as safe by the pharmaceutical industry and the various regulatory agencies. The Food and Drug Administration
(FDA) continues its longstanding approval for the use of aluminum adjuvant in vaccines, justified by various studies that found no adverse or long-term health effects due to aluminum adjuvant. ${ }^{3}$ The FDA concludes that "aluminum adjuvant containing vaccines have a demonstrated safety profile over six decades of use and have only uncommonly been associated with severe local reactions". ${ }^{5}$ In resemblance, the European Medicines Agency (EMA) concludes that "the use of aluminium hydroxide as adjuvant in products for specific immunotherapy is established for several decades and is regulated in the European Pharmacopoeia". ${ }^{6}$

\section{Aluminum as biological toxin}

Aluminum is a well demonstrated toxin in biological systems and its specific impacts on the nervous system have been widely documented. ${ }^{3}$ The Agency for Toxic Substances and Disease Registry (ATSDR) report that "there is a rather extensive database on the oral toxicity of aluminum in animals. These studies clearly identify the nervous system as the most sensitive target of aluminum toxicity".? Furthermore, a growing number of studies have linked the use of aluminum adjuvants to serious autoimmune outcomes in humans. ${ }^{3}$ In 2004, the FDA set a limit to no greater than $4-5 \mu \mathrm{g} / \mathrm{kg}$ bw/day for aluminum from parenteral sources for individuals with impaired kidney function and premature neonates, stating that levels above those have been associated with CNS and bone toxicity and that tissue loading may occur at even lower levels of administration. Newborn, infants and children up to 6 months of age in the U.S. and other developed countries receive 14.7 to 49 times more aluminum than the described FDA safety limit from mandatory immunization programs. ${ }^{3}$ The EMA restricted the aluminum content to $1.25 \mathrm{mg}$ per human dose. ${ }^{8}$ An aluminum-containing placebo is often used while evaluating safety and efficacy of vaccine clinical trials, either containing equal or greater amount of aluminum as to the test vaccine. Without exception, 
these trials shown a comparable rate of adverse reactions between the placebo and the test group. ${ }^{3}$ According to the FDA, a placebo is "an inactive pill, liquid, or powder that has no treatment value". ${ }^{9}$ The established neurotoxic properties of aluminum therefore suggest that aluminum-containing formulations cannot serve as a valid placebo. ${ }^{3}$

\section{Gulf war syndrome}

It must also be recognized that aluminum compounds may vary in their toxic potential depending on the specific route of administration. Mice fed with aluminum hydroxide at 66.5, 133 and 266mg Al $/ \mathrm{kg}$ bw /day did not reveal neuro developmental damage, while parenteral administration of aluminum chloride in rats at $40 \mathrm{mg} / \mathrm{kg}$ bw/day caused maternal deaths, embryo lethality, growth retardation and fetal abnormalities. It has been concluded that dietary aluminum is very poorly absorbed, $\sim 0.25 \%$, is absorbed into systemic circulation, and aluminum from vaccines may be absorbed at nearly $100 \% .^{3}$ A notable study linked Gulf War syndrome (GWS), a multisystem disorder, with multiple anthrax vaccinations administered over a short period of time. Injections of aluminum hydroxide in young CD-1 male mice at levels comparable to those administered to Gulf War veterans, were shown to cause significant motor neuron degeneration as well as impairments in motor function and decrements in spatial memory capacity. ${ }^{10}$

\section{Macrophagic myofasciitis}

A described syndrome termed macrophagic myofasciitis (MMF) has been specifically attributed to aluminum adjuvants in recipients of hepatitis A and B and tetanus toxoid (Td) vaccines. MFF patients suffer from diffuse arthromyalgias, chronic fatigue, muscle weakness and in some cases multiple sclerosis. It is characterized by muscle infiltration of granular positive macrophages and lymphocytes and inconspicuous muscle fiber damage. Electron microscopy and microanalysis showed the persistence of aluminum adjuvants at the site of injections and concomitant ongoing local immune reactions. Aluminum was shown to persist at the site of injection from several months up to 8years following vaccination. ${ }^{11}$ MMF lesions were subsequently also reproduced in rats by injection of aluminum. ${ }^{12}$ As with autism and GWS, MMF patients also show Th2 predominance and a significant risk factor in causing this syndrome may be aluminum hydroxide adjuvant. ${ }^{3}$

\section{Preclinical studies}

The EMA specifies that for non-clinical the toxicity of the adjuvant alone and the local tolerance e.g. inflammation should be studied depending on the route of administration. ${ }^{2}$ Further, the EMA concludes that "up to now, the spontaneous surveillance system of adverse events in pharmacovigilance and clinical studies have not revealed safety concerns regarding the aluminum in allergen immunotherapy apart from well-known local reactions (granuloma etc.). "Such granulosa are recognized in preclinical studies at the injection sites, usual subcutaneously. Granulomas do occur only if injections are repeated at the same site. The experience of one of the co-authors is that it takes at least 3 injections to cause such granuloma formation. They consist almost exclusively of an accumulation of enlarged macrophages with a pale, amphophilic-pinkish cytoplasm containing very tiny granules (Figure 1). These granulomas are surrounded by a thick fibrous capsule that causes the formation of abscesses (Figure 2). Similar lesions have been reported in animal models $s^{13,14}$ and in humans $s^{15,16}$ by others. There are reported cases of granuloma following tattooing with aluminum hydroxide containing inks (accidental inoculation). ${ }^{17}$ Except for granulomas at the injection site, other findings have not been observed in regulatory studies we evaluated. There were no indictors of neurotoxicity neither lesion in susceptible organs including the bone or immune system, nor indications for a general intoxication that could be related to exposure with aluminum hydroxide.

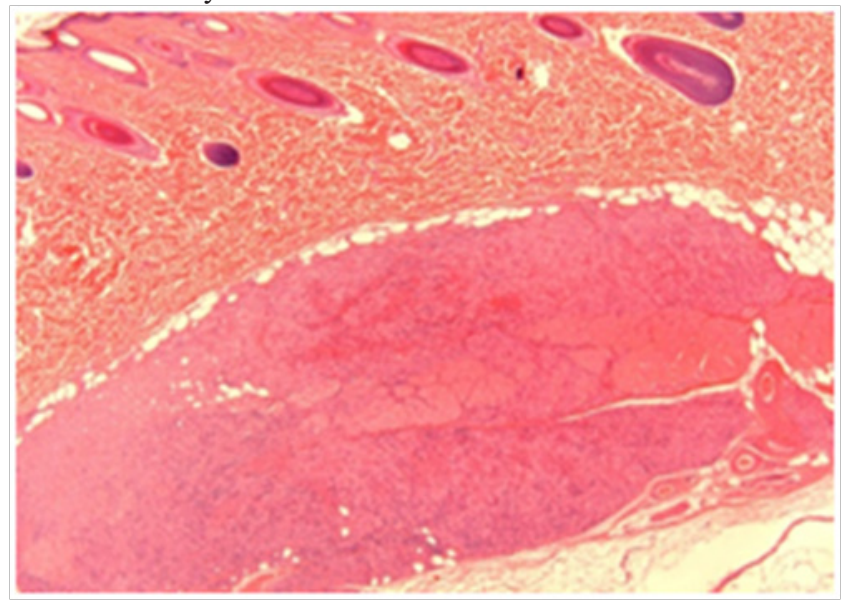

Figure I Cystic fibrous capsule surrounding aluminum hydroxide-induced granuloma forming subcutaneous abscess in a guinea pig after 14 days continuously daily injections of a vaccine containing aluminum hydroxide. Hematoxylin and eosin, lens $x 4$.

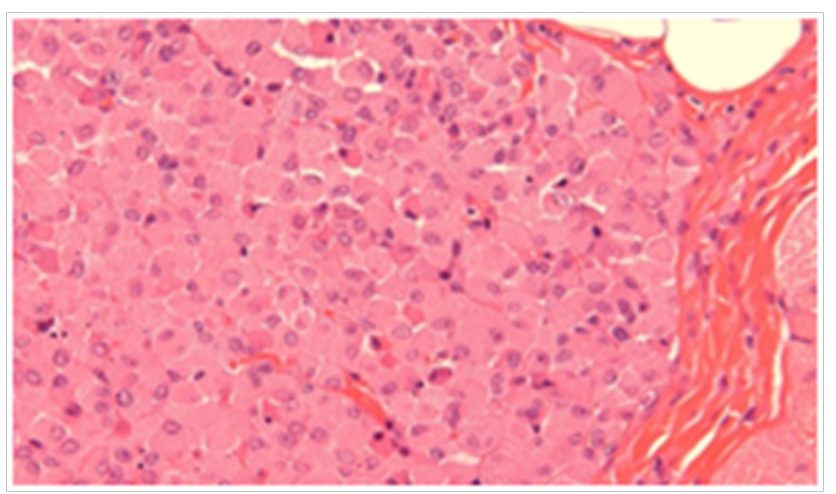

Figure 2 Enlarged macrophages with a pale cytoplasm containing tiny particles. From same study as in Figure I. Hematoxylin and eosin, lens x40.

\section{Advancing preclinical models}

Recently, a new technology allows tracing of aluminum hydroxide particles in tissue at very low levels and over a longer period by tagging aluminum adjuvant with fluorescent nano diamonds (AluDi). When injected in the tibial is anterior muscle of C57BL/6J mice, AluDia particles could be monitored in the brain tissue 3 weeks after injection..$^{18}$ Although no motoneuron death was observed in the mice model linking GWS to aluminum hydroxide, Shaw and Petrik observed a microglia and as trocytes reactivity in the spinal cord of CD1 mice together with an increase in anxiety, significant impairments in a number of motor functions and diminished spatial memory capacity. ${ }^{10}$ Progressive shrinkage of the local granuloma and rapid translocation of aluminum from the injection site to draining lymph nodes and spleen have been demonstrated..$^{18}$ But the long-term biodisposition of aluminum particles trapped in the local granuloma was previously mostly unexplored. Newly presented results show a delayed systemic translocation of aluminum adjuvant particles in CD-1 mice using AluDia particles. Granuloma remained for a longer time in the injected muscle despite progressive shrinkage from day 45 to day 270 
and delayed translocation of aluminum of the draining lymph nodes at the 270day endpoint was observed at injection of $400 \mu \mathrm{g} \mathrm{Al} / \mathrm{bw}$ $\mathrm{kg}$. Similar, translocation to the spleen was delayed. Remarkably the translocation of aluminum to the brain differs between the C57BL/6 J and $\mathrm{CD} 1$ mice. In contrast to $\mathrm{C} 57 \mathrm{Bl} / 6 \mathrm{~J}$ mice, no brain translocation by day 270 in CD1 mice neither increase aluminum cerebral content, nor behavioral changes were observed. ${ }^{19}$ It has previously been reported that the size of aluminum induced granuloma in rats is influenced by their genetic variety. In Lewis rats with Th1 biased immune response the granuloma has been demonstrated smaller compared to Sprague Dawley rats with balanced Th1/Th2 immunity. ${ }^{12}$ It can therefore not be excluded that the observed differences in biod is position may due to genetic strain differences. ${ }^{19}$ However, an additional experiment however confirmed early brain translocation at day 45 of aluminum injected subcutaneously at $200 \mu \mathrm{g} \mathrm{Al} / \mathrm{bw} \mathrm{kg}$ in CD-1 mice, which indicates that the translocation is dose dependent. Also, a higher rate of brain translocation after subcutaneous compared to intramuscular injection may be explained by a much higher density of dendritic cells with higher migrating properties in the skin. ${ }^{19}$

\section{Acknowledgments}

None.

\section{Conflicts of interest}

Author declares there are no conflicts of interest.

\section{Funding}

None.

\section{References}

1. Lindblad EB. Aluminium adjuvants--in retrospect and prospect. Vaccine. 2004;22(27-28):3658-3668.

2. The European Agency for the Evaluation of Medicinal Products Evaluation of Medicines for Human Use. Guideline on Adjuvants in Vaccines, UK. 2004. p.1-18.

3. Tomljenovic L, Shaw CA. Aluminum Vaccine Adjuvants: Are they Safe? Curr Med Chem. 2011;18(17):2630-2637.

4. Kool M, Soullié T, van Nimwegen M, et al. Alum adjuvant boosts adaptive immunity by inducing uric acid and activating inflammatory dendritic cells. J Exp Med. 2008;205(4): 869-882.
5. Vaccine Safety and Availability. US Food and Drug Administration, USA. 2004.

6. CHMP Safety Working Party's response to the PDCO regarding aluminium hydroxide contained in allergen products. The European Medicines Agency, UK. 2011. p.1-7.

7. Toxicological profile for aluminum. Agency for toxic substances and disease registry (ATSDR), USA. 2008. p.1-357.

8. Committee for Medicinal Products for Human Use. European Medicines Agency, UK. 2010.

9. Inside Clinical Trials: Testing Medical Products in People. US Food and Drug Administration, USA. 2009.

10. Shaw CA, Petrik MS. Aluminum hydroxide injections lead to motor deficits and motor neuron degeneration. J Inorg Biochem. 2009;103(11):1555-1562.

11. Authier FJ, Cherin P, Creange A, et al. Central nervous system disease in patients with macrophagic myofasciitis. Brain. 2001;124(Pt 5):974-983.

12. Authier FJ, Sauvat S, Christov C, et al. AlOH3-adjuvanted vaccineinduced macrophagic myofasciitis in rats is influenced by the genetic background. Neuromuscul Disord. 2006;16(5):347-352.

13. Valtulini S, Macchi C, Ballanti P, et al. Aluminium hydroxide-induced granulomas in pigs. Vaccine. 2005;23(30):3999-4004.

14. Goto N, Akama K. Histopathological studies of reactions in mice injected with aluminum-adsorbed tetanus toxoid. Microbiol Immunol . 1982;26(12):1121-1132.

15. Erdohazi M, Newman RL. Aluminium hydroxide granuloma. $\mathrm{Br}$ Med $\mathrm{J}$. 1971;3(5775):621-623.

16. Moss AL. Lessons in giving vaccines: soft tissue complications of vaccinations. J Public Health (Oxf). 2005;27(2):236-237.

17. McFadden N, Lyberg T, Hensten Pettersen A. Aluminum-induced granulomas in a tattoo. J Am Acad Dermatol. 1989;20(5 Pt 2):903-908.

18. Eidi H, David MO, Crépeaux G, et al. Fluorescent nanodiamonds as a relevant tag for the assessment of alum adjuvant particle biodisposition. BMC Med. 2015;13:144

19. Crépeaux G, Eidi H, David MO, et al. Highly delayed systemic translocation of aluminum-based adjuvant in CD1 mice following intramuscular injections. J Inorg Biochem. 2015;152:199-205. 